\title{
Minimal Device Encrustation on Vesair Intravesical Balloons in the Treatment of Stress Urinary Incontinence: Analysis of Balloons Removed from Women in the SOLECT Trial
}

\author{
Gommert A. van Koeveringe - Stefan De Wachter • Jack M. Zuckerman • \\ Giovanni Tommaselli · Michel J. de Wildt · Karel C. M. Everaert • \\ Dirk P. J. Michielsen · Jean-Jacques Wyndaele
}

Received: May 1, 2017 / Published online: June 9, 2017

(C) The Author(s) 2017. This article is an open access publication

\begin{abstract}
Introduction: Encrustation of urinary biomaterials is common; however, the incidence of surface deposition on the Vesair ${ }^{\circledR}$ intravesical pressure-attenuation balloon has not been previously reported. The purpose of this analysis is to determine the incidence and potential risk
\end{abstract}

Enhanced content To view enhanced content for this article go to http://www.medengine.com/Redeem/ 0998F06055555352.

G. A. van Koeveringe

Department of Urology, Maastricht University

Medical Centre, Maastricht, The Netherlands

S. De Wachter · J.-J. Wyndaele

University Hospital Antwerpen, University of

Antwerp-Urology, Antwerp, Belgium

J. M. Zuckerman ( $\square)$

Naval Medical Center San Diego, San Diego, CA,

USA

e-mail: jmzuckerman@gmail.com

G. Tommaselli

University Degli Studi Di Napoli "Federico II",

Naples, Italy

M. J. de Wildt

Catharina Ziekenhuis, Eindhoven, Netherlands

K. C. M. Everaert

Ghent University Hospital, Ghent, Belgium

D. P. J. Michielsen

UZBrussel-Urology, Brussels, Belgium factors for encrustation of the Vesair intravesical balloon.

Methods: The SOLECT trial is a prospective randomized controlled trial conducted at several European centers to evaluate the safety and efficacy of the Vesair balloon for the treatment of female stress urinary incontinence (SUI). Women included in the study demonstrated SUI symptoms for more than 12 months without complicating factors, such as history of recurrent urinary tract infections or nephrolithiasis. All balloons removed from women enrolled in the SOLECT trial were analyzed for surface characteristics and encrustation. Surface deposition severity was quantified and composition analyzed with infrared spectroscopy and scanning electron microscopy. Incidence of surface deposition was tabulated and risk factors analyzed.

Results: One hundred and five balloons removed from 75 women were included in this analysis. Measurable stone deposition of less than $1.5 \mathrm{~mm}$ was found on four balloons $(3.8 \%)$, surface granules were noted on 42 (40.0\%), surface film on $11(10.5 \%)$, and both granules and film on two (1.9\%). Analysis identified calcium oxalate both in measurable encrustation deposits as well as those with surface granulation. Pooled analysis found that dwell time was a risk factor for calcium deposition.

Conclusion: The rate of encrustation on the Vesair intravesical balloon is low and does not 
appear to increase the rate of adverse outcomes or reduce clinical efficacy.

Funding: Solace Therapeutics, Inc.

Keywords: Biomaterials; Calcium oxalate; Incidence; Urinary calculi; Urinary stress incontinence; Urology

\section{INTRODUCTION}

A multitude of biomaterials, defined as any substance that interfaces with host tissues, have been utilized in the urinary tract for centuries [1]. These urinary biomaterials started as primitive catheters made of metal tubes to treat urinary retention. Contemporary biomaterials have evolved into complex coated polymers that treat a variety of urinary tract pathology, such as synthetic urethral catheters, ureteral stents, and nephrostomy tubes.

Although a diversity of materials have been trialled over the years, current biomaterials used in the urinary tract include polyisoprene, polyethylene, polyvinylchloride, polyurethane, and silicone [2]. Each of these materials interacts with host tissues and its physiologic environment to differing degrees, a process termed biocompatibility. It is this biocompatibility, or lack thereof, which determines host tolerance, immunogenicity, degradation, and encrustation.

Other than patient tolerance, the primary concern of a chronic urinary biomaterial is infection and encrustation. Bacterial colonization of ureteral stents has been shown to occur in $28-90 \%$ of patients within a week after stent placement and urethral catheter colonization occurs just as quickly [3]. A subset of colonized biomaterials will then progress to true urinary tract infections (UTI). Additionally, bacterial colonization contributes to ureteral stent or other biomaterial encrustation through protein deposition and creation of a biofilm [4].

Vesair is a long-term, intravesical, polyurethane pressure-attenuating balloon that is being studied for the treatment of stress urinary incontinence (SUI). The balloon is an intravesical, free-floating device constructed of a thin polyurethane film (Fig. 1). Once placed into the

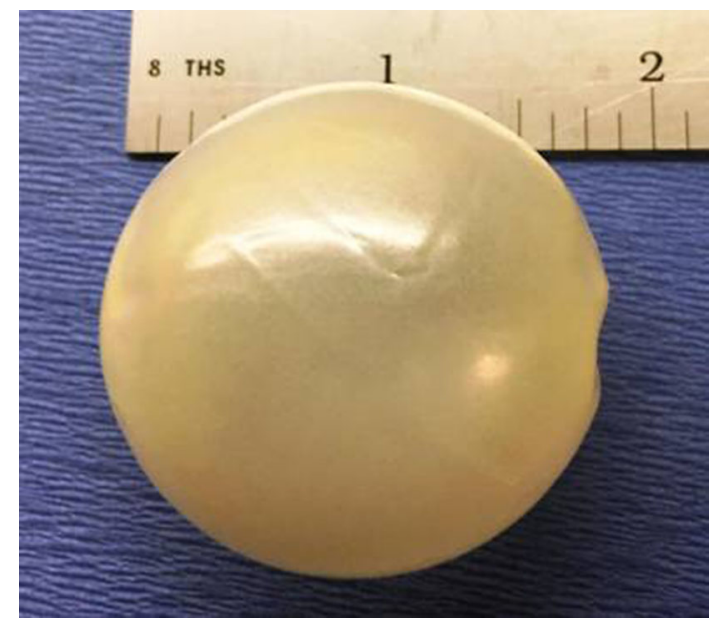

Fig. 1 Vesair balloon

bladder and inflated with $30 \mathrm{~mL}$ of air it floats to the bladder dome and away from the outlet. Its mechanism of action is based on the fact that urine in the bladder is relatively incompressible; therefore the pressure within the bladder increases when the surrounding abdominal pressure increases. In a susceptible patient, this intravesical pressure increase subsequently results in urinary incontinence by overcoming urethral resistance pressures [5]. Once indwelling, the Vesair balloon acts in accordance with Boyle's ideal gas law, which states that the pressure of a gas is inversely proportional to the volume $[6,7]$. The balloon compresses during transient increases in intravesical pressure, such as a cough or sneeze, thereby attenuating the pressure fluctuation similar to a "shock absorber" (Fig. 2).

Balloons are placed into the bladder via a transurethral approach. A Guardian Urethral Sheath $^{\circledR}$ is placed through the urethra to provide access to the bladder while protecting the urethra. A scope is placed through the sheath to perform diagnostic cystoscopy. The deflated balloon is then delivered via a 19-French delivery system through the Guardian Urethral Sheath ${ }^{\mathrm{TM}}$ and inflated with $0.7 \mathrm{~mL}$ of AirLoc ${ }^{\circledR}$ and $30 \mathrm{~mL}$ of air. Balloon removal is similarly conducted through the Guardian Urethral Sheath $^{\circledR}$ utilizing custom optical forceps to deflate and extract the balloon.

Two randomized controlled trials (RCT) have completed subject accrual and demonstrated 


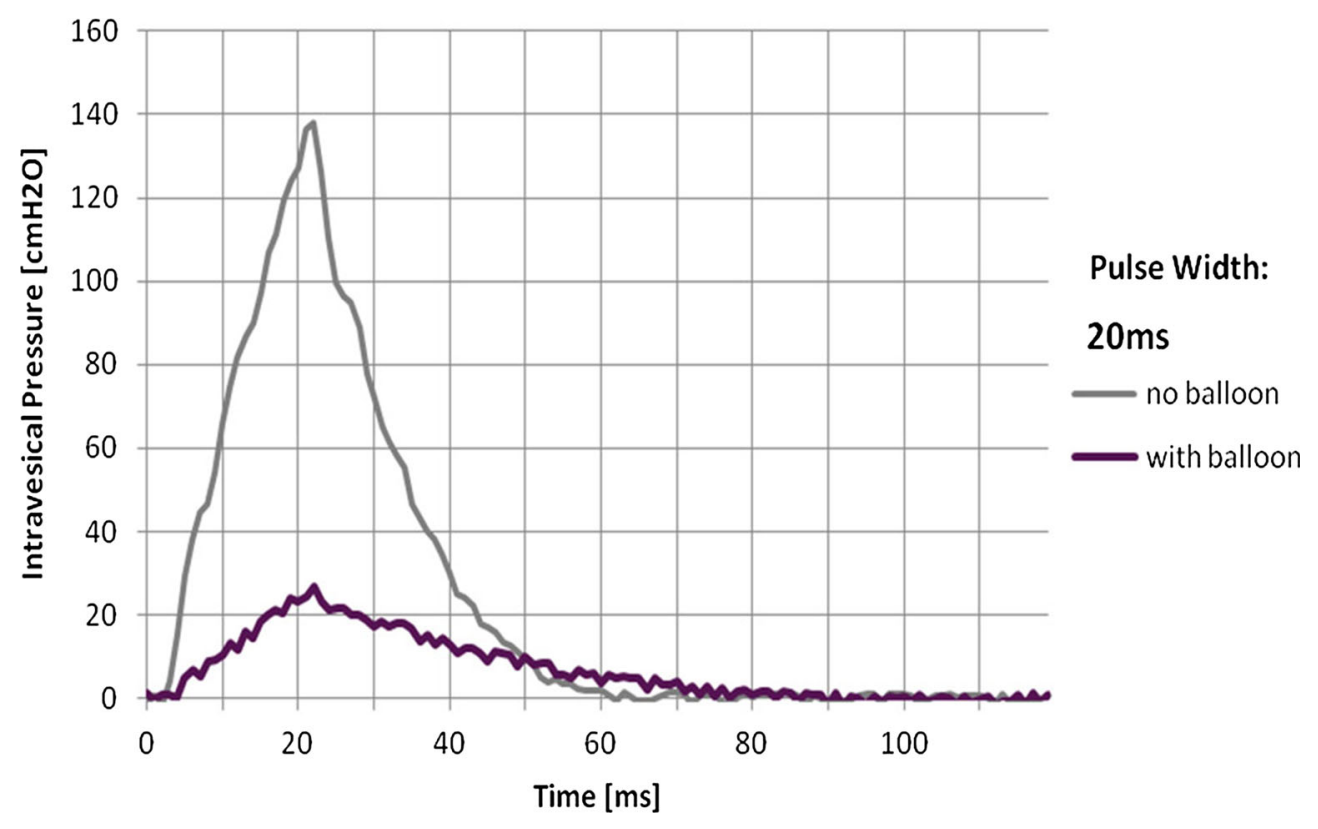

Fig. 2 Intravesical pressure attenuation

early success and patient satisfaction [8, 9]. Given the novelty of this new device, which is entirely intravesical and free floating, there is no published literature to draw on for rates of encrustation. Herein is a review of encrustation data on the current device that was used in the European SOLECT trial.

\section{METHODS}

The SOLECT trial is a prospective RCT conducted at several European centers to evaluate the safety and efficacy of the Vesair balloon for the treatment of female SUI [8]. Women were included if they had experienced SUI symptoms for more than 12 months, demonstrated SUI on either urodynamics or cough test, and had a Valsalva leak point pressure of at least $60 \mathrm{cmH}_{2} \mathrm{O}$. Participants were excluded in the setting of complicating factors such as a history of recurrent UTIs, nephrolithiasis, hematuria, concurrent pelvic radiation and pelvic organ prolapse. After the 3-month data collection, placebo patients were unblinded and offered to crossover and receive a balloon if desired. Contrary to the previous US trial where balloons were exchanged per protocol every 3 months, balloons in the SOLECT trial were allowed to remain indwelling for up to 1 year prior to exchange.

All procedures followed were in accordance with the ethical standards of the responsible committee on human experimentation (institutional and national) and with the Helsinki Declaration of 1964, as revised in 2013. Informed consent was obtained from all patients for being included in the study. Ethics committee approvals were obtained in Belgium $(12 / 27 / 228)$, Italy $(95 / 12)$, and the Netherlands (AZM: 12-1-043). The first randomized trial for this device utilized a "seamed" pressure-attenuation balloon, which resembled the original device prototype. The seam on this device was thought to contribute to potential stone formation given its rough surface. The balloon has since been modified from its original design and is currently a seamless, smooth, spherical balloon constructed of thin medical grade polyurethane (Fig. 1). The valve, which previously protruded from the balloon, is now welded flush with the device to limit uneven surfaces that may contribute to bladder irritation and stone formation. It is this updated Vesair 
balloon design that was reported in the 3-month results from the SOLECT trial and what was analyzed for this study.

All balloons removed as part of the SOLECT trial, regardless of indication for removal, were saved in a specimen collection container and submitted for analysis. A central laboratory at Solace Therapeutics evaluated each balloon for device coloration, degradation, and encrustation utilizing a Nikon measuring microscope (model MM-400/SL) An inspection protocol and quality control procedures were followed. Encrustation was visually quantified by measuring the depth of the thickest portion of deposition on each balloon. A 10-point scale was created to stratify encrustation severity ranging from $0(0-0.1 \mathrm{~mm})$ up to $10(>9.5 \mathrm{~mm})$ as outlined in Fig. 3 [9]. Fourier transform infrared spectroscopy analysis was performed on representative balloons with sufficient deposit to determine the stone composition. Additionally, notation was made for balloons with surface changes, but no stone formation, specifically surface granulation or a surface film. A selection of balloons that appeared to have a surface film were sent for scanning electron microscopy (SEM) and DNA sequencing analysis to determine the presence of bacteria in what was presumed to be a biofilm. Finally, bacteriologic data was tabulated from all urine cultures for patients diagnosed with a UTI. The primary

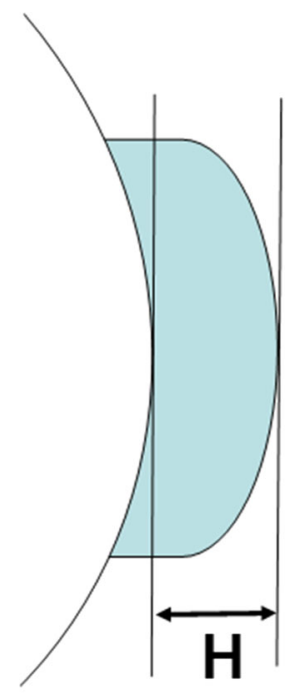

\begin{tabular}{|c|c|}
\hline $\mathrm{H}(\mathrm{mm})$ & Score \\
\hline $0-0.1$ & 0 \\
\hline $0.1-1.49$ & 1 \\
\hline $1.5-2.49$ & 2 \\
\hline $2.5-3.49$ & 3 \\
\hline $3.5-4.49$ & 4 \\
\hline $4.5-5.49$ & 5 \\
\hline $5.5-6.49$ & 6 \\
\hline $6.5-7.49$ & 7 \\
\hline $7.5-8.49$ & 8 \\
\hline $8.5-9.49$ & 9 \\
\hline$>9.5$ & 10 \\
\hline
\end{tabular}

Fig. 3 Encrustation scoring system aim of the present study is to describe the incidence, severity, and risk factors for encrustation on Vesair intravesical balloons.

\section{RESULTS}

Seventy-seven women had Vesair balloons placed in conjunction with the SOLECT trial. Mean age at time of implantation was 48.9 years with a mean body mass index of $26.1 \mathrm{~kg} / \mathrm{m}^{2}$. All but one subject was Caucasian, with the single subject's race being unclassified. Despite a predominantly middle and older aged population of women, $53.3 \%$ of participants were premenopausal and 20\% perimenopausal. Most women were non-smokers with only $16.0 \%$ admitting to active tobacco use, averaging one pack per day.

In this cohort of 77 women, 108 Vesair balloons were placed; all but three balloons from two patients were returned to the central laboratory for full evaluation. The median dwell time for all balloons was 154 days (range of $4-441$, interquartile range of 286 or \pm 143 days). A proportion of subjects underwent balloon exchanges during the follow-up period, including 14 women who received two balloons, five with three balloons, and a single patient each with five and seven balloon exchanges. The remaining 56 women had a single balloon placed.

Of the 105 balloons analyzed after explantation only four $(3.8 \%)$ had measurable stone deposition, each with an encrustation scale score of 1 (stone deposit 0.1-1.49 mm) (Fig. 3). The surface area of the deposition on these four was less than $1 \mathrm{~mm}^{2}$ on three of the balloons and $40 \mathrm{~mm}^{2}$ on one. These four balloons had been indwelling for an average 302 days (93, $322,383,412$ days). None of the four subjects with encrustation scale score of 1 was diagnosed with a UTI and none complained of gross hematuria or dysuria/bladder irritation. Each of these four women had successful outcomes from their Vesair balloon by SOLECT trial criteria (three composite success and one by pad weight only). The surface deposits from each of these four balloons were determined to be calcium oxalate on spectroscopic analysis. 
In balloons without measurable surface deposition, surface granulation was identified on $42(40 \%)$, surface film on $11(10.5 \%)$, and both granulation and film on two (1.9\%). Forty-six balloons $(43.8 \%)$ had no significant surface changes at the time of explantation. Calcium oxalate composed the surface granules on SEM; however, no calcium was noted on SEM analysis for balloons with surface film alone. DNA sequencing on the surface films found the presence of Acinetobacter baumannii and Micrococcus luteus. No Escherichia coli, Proteus mirabilis, or other common urinary tract pathogens were noted in any surface films. Figure 4 shows representative photographs of each surface deposit category.

Balloon surface characteristics and dwell times are depicted in Table 1. Balloons with encrustation or surface granulation were indwelling for a median of 352.5 days (range 4-412 days) or 201 days (range 7-441, intraquartile range $290 \pm 145$ days), respectively, whereas balloons with no surface changes were removed at a median of 105 days (range $4-419$, intraquartile range 238 or \pm 119 days). Though not individually significant, combining balloon groups found that the 48 balloons with any degree of surface calcium deposition (i.e., measurable encrustation or surface granulation) had a significantly longer median dwell time of 216 days (range 7-441 days, intraquartile range 286.5 or \pm 143.2 days) versus a median of 107 days (range $4-149$ days, intraquartile range 210 or \pm 105 days) for the 57 balloons without any surface deposition $(p=0.017)$. Similar analysis for subject age did not reveal any statistical significance.

A UTI was diagnosed in 11 subjects (14.3\%) on average 153 days following balloon placement (median 123 days, range 7-359 days). Of those, only six were culture positive with the other five diagnosed on the basis of symptoms and/or urinalysis with a negative culture. The most common solitary bacteria grown in culture was E. coli in two specimens, followed by Streptococcus agalactiae in a single culture, and Staphylococcus aureus and Enterococcus faecalis in the same specimen. Two additional cultures had mixed bacterial growth. None of the subjects diagnosed with a UTI developed measurable encrustation, though all but two had surface granulation, film, or both. The majority of these subjects diagnosed with a UTI kept the balloon indwelling through treatment. Two underwent balloon removal at the time of UTI diagnosis, one of whom had a balloon subsequently replaced after adequate UTI treatment.

\section{DISCUSSION}

Encrustation on any urinary biomaterial results from ionic constituents in the urine crystallizing on its surface and becoming incorporated into the device biofilm. This biomaterial encrustation may be divided into two categories, as the mechanism of formation is slightly different.

The most common type of biomaterial encrustation is that associated with a UTI, specifically an infection with urease-producing bacteria. This mechanism of encrustation is most commonly seen with indwelling urethral catheters. Bacterial spread occurs from the urethral meatus and proceeds up the exterior or interior of the catheter into the bladder. Biomaterial colonization can occur even in the presence of a negative urine culture and often within the first 7 days from catheter insertion [10]. When the colonization is with a urease-producing organism, most frequently $P$. mirabilis, urea is cleaved to eventually form carbon dioxide, which raises the urinary $\mathrm{pH}$ and decreases both calcium phosphate and struvite solubility, leading to stone formation [11].

The second type of encrustation is that which is seen on ureteral stents. Internal stents are less commonly colonized with $P$. mirabilis and instead a non-urease-producing bacterium, such as E.coli, is more often found in a stent bacterial biofilm [12]. Biofilm on ureteral stents has been shown to promote encrustation by attracting and incorporating crystals on its surface. However, in the absence of significant $\mathrm{pH}$ changes associated with this non-urease colonization, stone deposition is driven by the same factors associated with nephrolithiasis, such as hypocitraturia, hypercalciuria, and hyperoxaluria, and stones are more often composed of calcium oxalate $[11,13]$. 

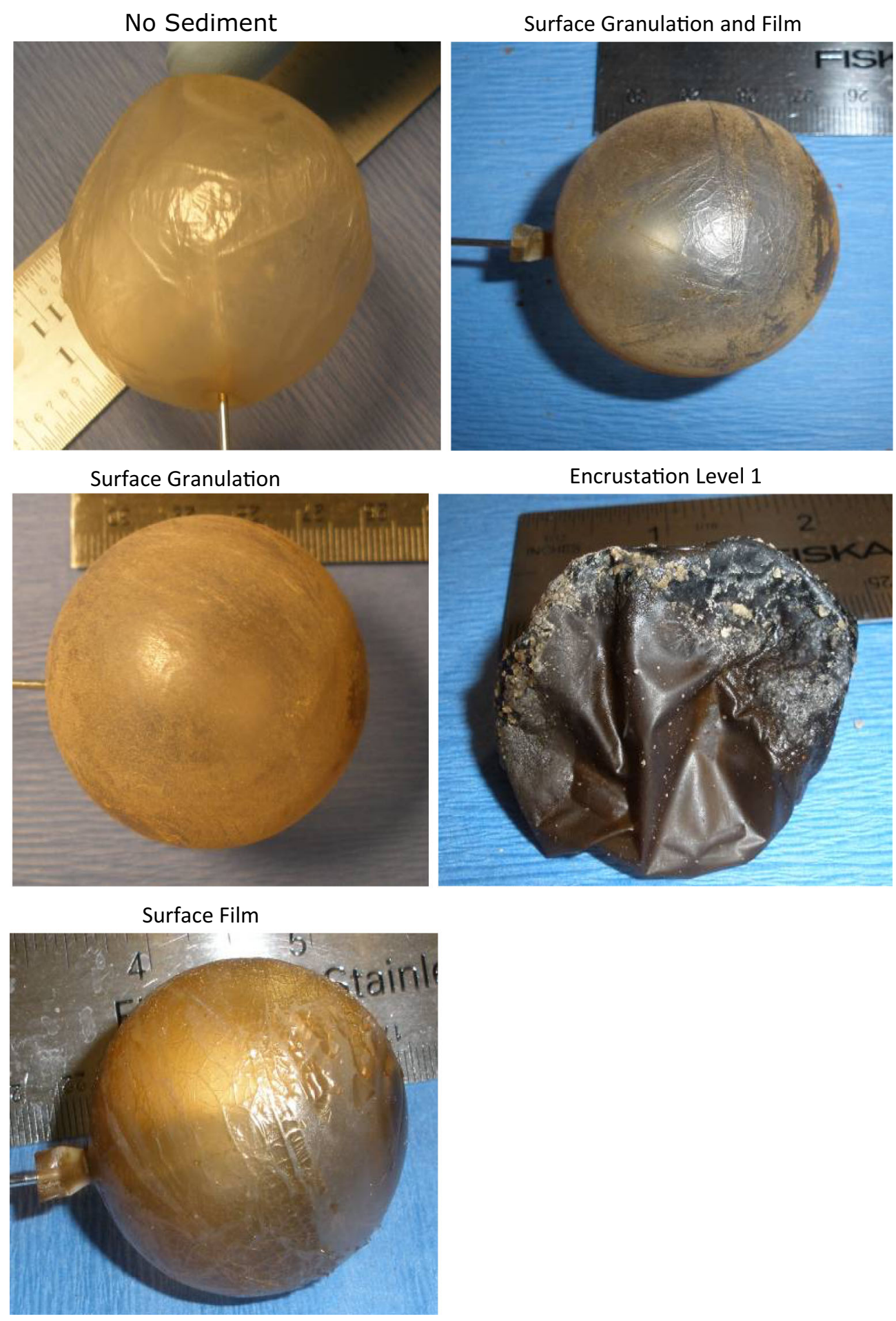

Fig. 4 Photographs of balloons demonstrating each of the surface deposit categories noted

It has been shown in this analysis that the rate of encrustation of Vesair intravesical balloons is minimal and tolerable. In no cases did the degree of encrustation lead to complications or difficulty removing the device when necessary. Additionally, subjects with the most 


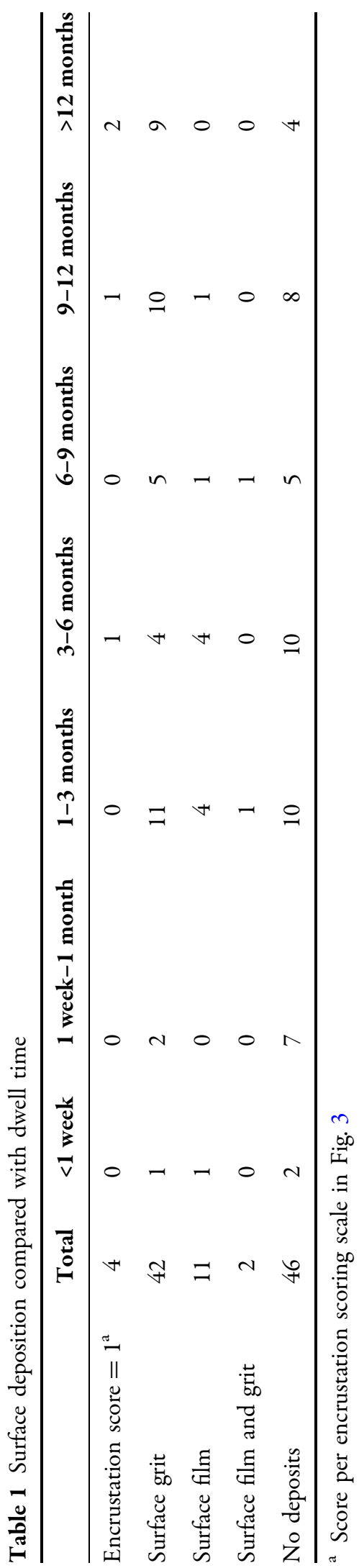

severely encrusted balloons each had successful SUI outcomes despite the encrustation, and none were diagnosed with a UTI.

Mineralogical analysis found calcium oxalate on both balloons with surface granulation and the few with measurable stone deposition. Although the Vesair balloon is a chronic intravesical biomaterial, similar to a urethral catheter, the fact that there were no struvite or calcium phosphate stones suggests an alternate mechanism of stone deposition on this device. Lack of a direct external connection to allow rapid bacterial colonization may limit susceptibility of the Vesair balloon to this type of encrustation.

Calcium oxalate deposition on urinary biomaterials is much more commonly seen with indwelling ureteral stents. The incidence and rate of stone formation on ureteral stents, however, are much higher than what was found in the present study. Kawahara and colleagues analyzed 330 ureteral stents after removal and noted a nearly $50 \%$ rate of encrustation at a mean dwell time of only 52.8 days [14]. Severe encrustation, defined as inability to advance a stiff wire through the stent, was seen in $15.8 \%$ of stents removed. Dwell time was found to be a strong risk factor for stent encrustation, incrustation, and resistance to stent removal.

In addition to stent dwell time, other authors have suggested additional risk factors for ureteral stent encrustation. Sighinolfi and associates suggested different etiologies for stone deposition on the proximal versus distal coil of ureteral stents [13]. On the distal coil, a location more similar to that of the Vesair balloon, the presence of a UTI and advanced patient age contributed to stone formation. Advanced age may be a surrogate for an elevated post-void residual, which is a known risk factor for bladder stones and therefore would also lead to distal ureteral stent calcification.

Compared with indwelling ureteral stents, the rate of encrustation of the Vesair balloon in this study is remarkably low. This is despite the fact that the Vesair balloon is made of polyurethane, the same polymeric biomaterial used in most modern ureteral stents [15]. Additionally, Vesair balloons in this study were left indwelling for a longer duration (mean 
182 days) than would be common for most ureteral stents. There are a variety of theoretical explanations for this difference. This study population excluded women who might be at high risk for stone formation, such as those with a history of nephrolithiasis and recurrent UTIs. Contrastingly, it is likely those exact risk factors that were the indication for stent placement in a majority of patients included in ureteral stent encrustation trials. Other than patient selection, however, the Vesair balloon has inherent properties that separate it from both ureteral stents and indwelling urethral catheters to reduce the risk of stone formation. The surface of the Vesair balloon is almost entirely smooth, with very few interstices that allow stone deposition to initiate. The balloon surface is elastic and can stretch and contract as a result of external forces placed upon it. It is also a freely mobile balloon, which floats around the bladder in continually different locations depending on patient positioning, urine volume, and activity. Compared with ureteral or urethral catheters, which are both anchored in place, the mobility and elasticity of the Vesair balloon likely limit the ability of surface deposition to begin and/or propagate.

There are several limitations to this study. While $97 \%$ of removed balloons were sent to the central laboratory, three balloons were not recovered. If any of these had significant stone deposition and had been captured it could significantly alter our results. Balloon removal is performed under direct vision as has previously been described $[8,9]$. It is possible, however, that in the process of removing a balloon, encrustation that had been loosely adherent could have been detached and therefore would not be accounted for in the data analysis. Additionally, the surface characteristics of the balloon (as a result of the inherent variability of the manufacturing processes) and its delivery out of the catheter could contribute to the variability of the data measured. While all balloons passed manufacturing inspection for smooth surface characteristics, the surface characteristics of the balloon were not evaluated after removal in this analysis. Finally, bacterial contamination as a cause for the unusual bacteria noted in our DNA analysis of the balloon surface film cannot be ruled out. While care was taken to place each balloon into the collection container after removal, the sterility of these containers cannot be guaranteed and additional contamination may have occurred during balloon removal and transport. Limitations notwithstanding, the authors feel confident that the rate of encrustation on Vesair balloons in this cohort is comparatively low and does not appear to lead to adverse patient outcomes or device efficacy.

\section{CONCLUSIONS}

The rate of encrustation on the Vesair intravesical pressure-attenuating balloon is low and does not appear to increase the rate of adverse outcomes or reduce clinical efficacy. The mechanism of stone deposition appears more consistent with what is seen with indwelling ureteral stents; however, unique properties of the Vesair balloon seem to limit the ability of crystals to deposit on the balloon surface.

\section{ACKNOWLEDGEMENTS}

This study and this journal's article processing charges and Open Access fee were funded by Solace Therapeutics. In addition to the named authors, the following clinicians participated in the study: Carmen Formisano and Annamaria Fabozzi, Universita Degli Studi Di Napoli "Federico" II, Naples, Italy; Dennis Oerlemans and Marc Paffen, Maastricht University Hospital, Maastricht, the Netherlands; Anne-Francoise Spinoit and Christel Van Camp, Ghent University Hospital, Ghent, Belgium; Evert Koldewijn and Wout Scheepens, Catharina Ziekenhuis, Eindhoven, the Netherlands; Roberto Angioli, Marzio Angelo Zullo, Paolo Gennari, Alessia Aloisi, Roberto Montera, and Francesco Plotti, Universita di Roma Campus Biomedico, Rome, Italy. All named authors meet the International Committee of Medical Journal Editors (ICMJE) criteria for authorship for this manuscript, take responsibility for the integrity of the work as a whole, and have given final approval for the version to be published. All authors had full 
access to all of the data in this study and take complete responsibility for the integrity of the data and accuracy of the data analysis.

Disclosures. Gommert A. van Koeveringe, Stefan De Wachter, Giovanni Tommaselli, Michel J. de Wildt, Karel C. M. Everaert, Dirk P. J. Michielsen, and Jean-Jacques Wyndaele enrolled patients in this study that was funded by Solace Therapeutics. Gommert A. van Koeveringe, Stefan De Wachter, and Giovanni Tommaselli have received speaker support from Solace Therapeutics, Inc. Giovanni Tommaselli was a consultant to Johnson and Johnson.

Compliance with Ethics Guidelines. All procedures followed were in accordance with the ethical standards of the responsible committee on human experimentation (institutional and national) and with the Helsinki Declaration of 1964, as revised in 2013. Informed consent was obtained from all patients for being included in the study.

Data Availability. The datasets generated during and/or analyzed during the current study are available from the corresponding author on reasonable request.

Open Access. This article is distributed under the terms of the Creative Commons Attribution-NonCommercial 4.0 International License (http://creativecommons.org/licenses/ by-nc/4.0/), which permits any noncommercial use, distribution, and reproduction in any medium, provided you give appropriate credit to the original author(s) and the source, provide a link to the Creative Commons license, and indicate if changes were made.

\section{REFERENCES}

1. Mardis HK, Kroeger RM, Morton JJ, Donovan JM. Comparative evaluation of materials used for internal ureteral stents. J Endourol. 1993;7:105-15.

2. Beiko DT, Knudsen BE, Watterson JD, Cadieux PA, Reid G, Denstedt JD. Urinary tract biomaterials. J Urol. 2004;171:2438-44.
3. Ben-Meir D, Golan S, Ehrlich Y, Livne PM. Characteristics and clinical significance of bacterial colonization of ureteral double-J stents in children. J Pediatr Urol. 2009;5:355-8.

4. Elves AW, Feneley RC. Long-term urethral catheterization and the urine-biomaterial interface. Br J Urol. 1997;80:1-5.

5. Rovner ES, Ginsberg DA, Raz S. The UCLA surgical approach to sphincteric incontinence in women. World J Urol. 1997;15(5):280-94.

6. Walski TM, Haestad Methods. Advanced water distribution modeling and management. 1st ed. Waterbury: Haestead; 2003.

7. Wylie EB, Streeter VL, Suo L. Fluid transients in systems. Englewood Cliffs: Prentice Hall; 1993.

8. Wyndaele JJ, De Wachter S, Tommaselli GA, et al. A randomized, controlled clinical trial of an intravesical pressure-attenuation balloon system for the treatment of stress urinary incontinence in females. Neurourol Urodyn. 2015;35:252-9.

9. Rovner ES, Dmochowski RR, Leach GE, Jayne C, Snyder JA. A randomized, controlled clinical trial of a novel intravesical pressure attenuation device for the treatment of stress urinary incontinence. J Urol. 2013;190:2243-50.

10. Nickel JC, Grant SK, Costerton JW. Catheter-associated bacteriuria. An experimental study. Urology. 1985;26:369-75.

11. Shaw GL, Choong SK, Fry C. Encrustation of biomaterials in the urinary tract. Urol Res. 2005;33:17-22.

12. Keane PF, Bonner MC, Johnston SR, Zafar A, Gorman SP. Characterization of biofilm and encrustation on ureteric stents in vivo. $\mathrm{Br} \mathrm{J}$ Urol. 1994;73:687-91.

13. Sighinolfi MC, Sighinolfi GP, Galli E, et al. Chemical and mineralogical analysis of ureteral stent encrustation and associated risk factors. Urology. 2015;86:703-6.

14. Kawahara T, Ito H, Terao H, Yoshida M, Matsuzaki J. Ureteral stent encrustation, incrustation, and coloring: morbidity related to indwelling times. J Endourol. 2012;26:178-82.

15. Chew BH, Denstedt JD. Technology insight: novel ureteral stent materials and designs. Nat Clin Pract Urol. 2004;1:44-8. 